05

\title{
Электрохимическая эмиссия при деформировании и разрушении алюминий-магниевого сплава в водной среде
}

\author{
(C) А.А. Шибков, М.Ф. Гасанов, А.Е. Золотов, М.А. Желтов, А.А. Денисов, Р.Ю. Кольцов, С.С. Кочегаров \\ Тамбовский государственный университет им. Г.Р. Державина, \\ 392000 Тамбов, Россия \\ e- mail: shibkov@tsu.tmb.ru
}

Поступило в Редакцию 4 апреля 2019 г.

В окончательной редакции 4 апреля 2019 г.

Принято к публикации 5 июня 2019 г.

Пространственно-временные структуры деформационных полос Портевена-Ле Шателье на стадии образования шейки и разрушения алюминий-магниевого сплава, деформируемого в водной среде, исследовали комплексом in situ методов, включающим высокоскоростную видеосъемку поверхности и метод электрохимической эмиссии. Последний основан на измерении и анализе скачков электродного потенциала деформируемого металла в условиях проявления прерывистой деформации. Установлено, что дискретные сигналы электрохимической эмиссии в полосе частот $10 \mathrm{~Hz}-10 \mathrm{kHz}$ содержат информацию о количестве деформационных полос, моментах зарождения и стадиях их роста, о статистической временной структуре полос и т.д. Выявлена характерная серия сигналов на стадии предразрушения - электрохимический предвестник формирования шейки и разрушения образца. Обсуждена связь генерации сигналов электрохимической эмиссии с локализацией пластической деформации и разрушением оксидной пленки на поверхности алюминиевого сплава, контактирующего с водной средой.

Ключевые слова: эффект Портевена-Ле Шателье, алюминий-магниевый сплав, электрохимическая эмиссия, полоса деформации, разрушение.

DOI: 10.21883/JTF.2020.01.48666.151-19

\section{Введение}

Согласно современным представлениям, вязкое разрушение металлов имеет дислокационную природу: на всех масштабных уровнях ему предшествует пространственно-временная локализация пластической деформации от заторможенного скопления нескольких десятков дислокаций до полос макролокализованной деформации на завершающей стадии образования шейки перед макроразрушением. Это подтверждает основополагающее представление, сформулированное А.В. Степановым [1] о том, что разрушению всегда предшествует пластическая деформация. Присущая пластической деформации неустойчивость (прерывистость) и неоднородность (локализация) обусловливает сложную иерархию структурных уровней деформации и связанную с ней многомасштабность процесса вязкого разрушения.

На макроскопическом структурном уровне разрушению металла предшествует формирование полос макролокализованной деформации, а затем шейки - глобальной потери устойчивости материала перед развитием магистральной трещины. Это прежде всего относится к металлам, деформируемым при температурах порядка и выше дебаевской [2,3]. Роль макрополос в образовании шейки перед разрушением подробно рассмотрена в работах томской школы физиков-прочнистов [4-6] на примере ряда металлических сплавов $(\mathrm{Ni}-\mathrm{Ti}, \alpha-\mathrm{Fe}$, Al, сталь ВК1-12Ті и др.). Вместе с тем многие авиационные алюминиевые сплавы демонстрируют преры- вистую деформацию Портевена-Ле Шателье (ПЛШ) и полосообразование при комнатной и повышенных температурах, когда полосы распространяются задолго до разрушения, сопровождая деформирование металлического сплава после полосы Людерса. Разрушение таких сплавов всегда происходит по деформационной полосе. Связь полос ПЛШ с разрушением представляет малоисследованную проблему физического материаловедения, которой посвящено небольшое количество публикаций [7-9]. В настоящей работе исследуется эта связь на основе статистического и корреляционного анализа данных скоростной видеосъемки распространяющихся деформационных полос и магистральной трещины и сопутствующих временных рядов - прерывистых деформационных кривых и сигналов электрохимической эмиссии - скачков электродного потенциала, связанных с формированием деформационных полос в алюминиймагниевом сплаве, деформируемом в водной среде.

\section{1. Материал и методика исследований}

Материалом исследования служил алюминий-магниевый сплав АМг6 (Al-6.15 wt.\% Mg-0.65 wt.\% Mn-0.25 wt.\% Si-0.12 wt.\% Zn-0.2 wt.\% Fe-0.1 wt.\% Cu), демонстрирующий эффект ПЛШ при комнатной температуре. Образцы в форме двухсторонних лопаток с размером рабочей части $6 \times 3 \times 0.5 \mathrm{~mm}$ вырезали машинным способом из листового проката после холодной прокатки, отжигали в течение $1 \mathrm{~h}$ при температуре $450^{\circ} \mathrm{C}$ и закаливали 
на воздухе. Затем образцы, погруженные в дистиллированную воду (ГОСТ 6709-72, удельное сопротивление $\left.2 \cdot 10^{5} \Omega \cdot \mathrm{cm}\right)$, растягивали с постоянной скоростью $\dot{\varepsilon}_{0}=3 \cdot 10^{-3} \mathrm{~s}^{-1}$ в испытательной машине Instron (модель 3344) при комнатной температуре. Методика измерения скачков электродного потенциала $\Delta E-$ сигналов электрохимической эмиссии (ЭХЭ), - с помощью хлорсеребряного электрода сравнения (ЭСР-10101) и импульсного предусилителя в полосе частот $10 \mathrm{~Hz}-10 \mathrm{kHz}$ изложена в работе [10]. Динамику деформационных полос ПЛШ исследовали с помощью съемки цифровой видеокамерой фирмы Photron (FASTCAM Mini UX50/100). Скорость съемки варьировали от 50 до 20000 frames/s в зависимости от задачи исследования (видеосъемка всех полос ПЛШ для построения пространственной гистограммы полос, кинетика отдельной полосы, эволюция стадии предразрушения и т.д.)

\section{2. Результаты и обсуждение}

\section{1. Корреляция сигналов ЭХЭ с прерывистой деформацией ПЛШ и полосообразованием}

На рис. 1 представлена прерывистая часть кривой растяжения от критической деформации $\varepsilon_{c}$ появления первого деформационного скачка до разрушения сплава АМг6 и соответствующий электрохимический отклик дискретные сигналы электрохимической эмиссии $\Delta E(t)$. В течение всего времени деформирования вели видеосъемку поверхности образца со скоростью $50 \mathrm{frames} / \mathrm{s}$. Установлено, как и в работе [11], что каждому дискретному сигналу ЭХЭ соответствует формирование полосы макролокализованной деформации. Это дает возможность строить пространственно-временное распределение источников ЭХЭ, в частности, выделять сеты сигналов ЭХЭ, генерируемых деформационными полосами, проходящими через заданное сечение, особенно сечение, через которое пройдет магистральная трещина. На рис. 1 такой сет сигналов ЭХЭ отмечен треугольными точками. В силу важности для темы настоящего исследования будем называть его „магистральным“ сетом сигналов ЭХЭ, а соответствующее сечение - „магистральным“.

Следует отметить, что в структуре дискретных сигналов ЭХЭ можно выделить кластеры скоррелированных сигналов (рис. 1, кривая 2), в которых наблюдается приблизительно линейная зависимость между амплитудой сигнала ЭХЭ $\Delta E_{m}$ и последующей паузой $\tau$ : $\Delta E_{m}=C\left(\tau-\tau_{0}\right)$, где $C-$ коэффициент пропорциональности, который увеличивается с ростом номера кластера в пределах $8-12 \mathrm{mV} / \mathrm{s}$, а $\tau_{0} \approx 0.35 \mathrm{~s}-$ минимальная пауза между сигналами ЭХЭ и соответственно между скачками напряжения при заданной скорости деформации (рис. 2), связанная с механизмом деформационного старения дислокаций. Кластеры сигналов ЭХЭ разделены относительно большими паузами, которые не укладываются в зависимость $\Delta E_{m}(\tau)$ внутри кластеров.

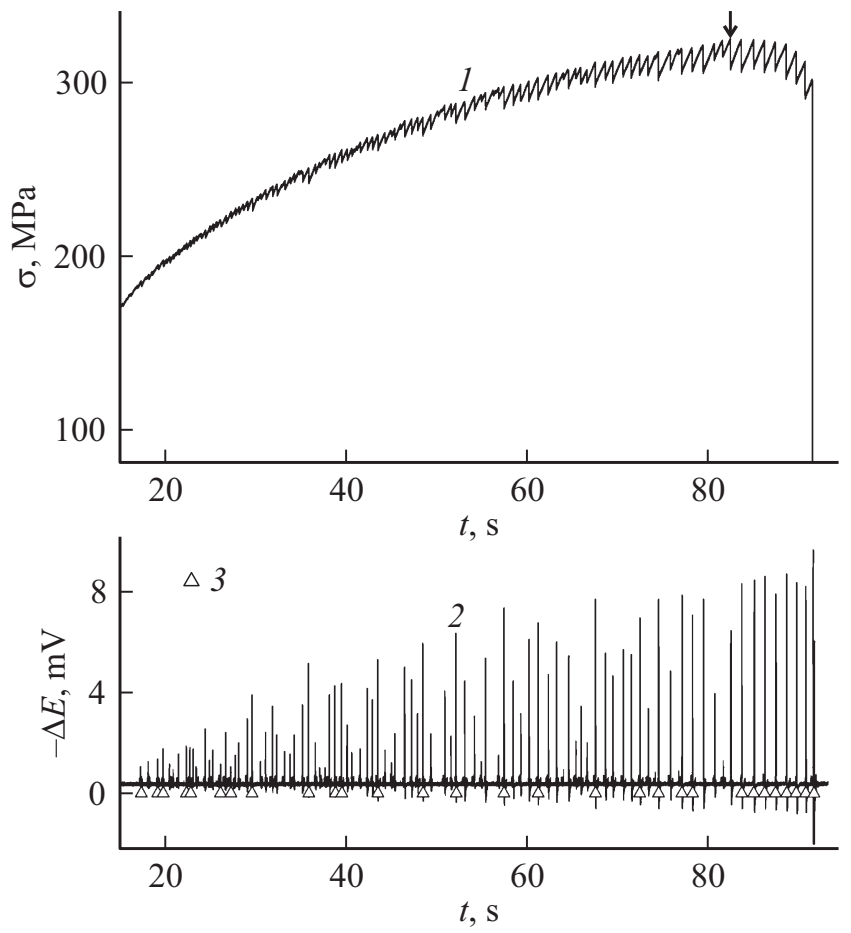

Рис. 1. Прерывистая кривая деформации в координатах $\sigma-t(1)$ и сигналы ЭХЭ $\Delta E(2)$ при растяжении сплава АМг6 со скоростью $\dot{\varepsilon}_{0}=3 \cdot 10^{-3} \mathrm{~s}^{-1}$. „Магистральный“ сет сигналов ЭХЭ (3) отмечен треугольниками. Стрелкой отмечен момент достижения максимальной нагрузки $F_{\max }$.

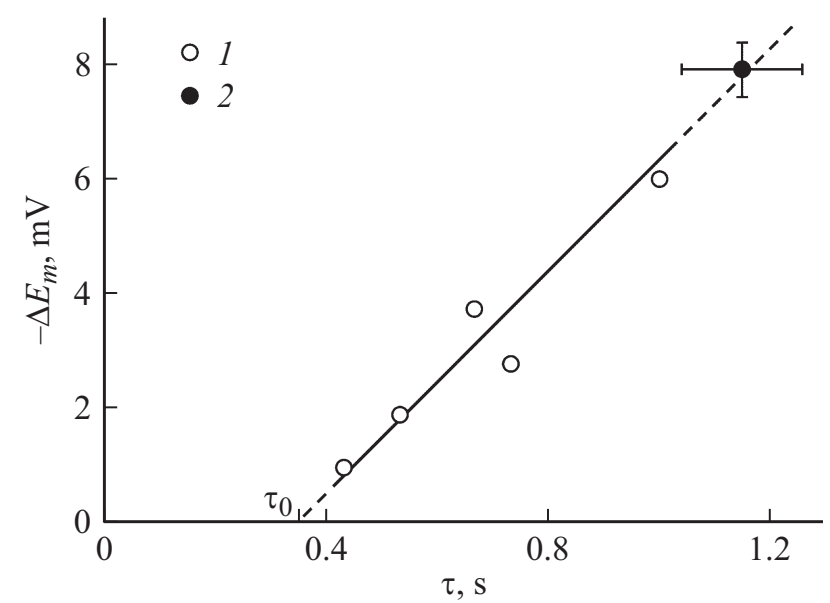

Рис. 2. Зависимость амплитуды сигнала ЭХЭ $\Delta E_{m}$ от последующей паузы $\tau$ между сигналами: 1 - для типичного кластера сигналов ЭХЭ в средней части прерывистой кривой растяжения; 2 - для последнего кластера сигналов, зарегистрированных после достижения максимальной нагрузки, $F_{\max }$. $\tau_{0} \approx 0.35 \mathrm{~s}-$ минимальная пауза между дискретными сигналами ЭХЭ.

Важно подчеркнуть, что один или два высокоамплитудных сигнала ЭХЭ в каждом таком кластере принадлежат магистральному сету сигналов ЭХЭ, а в последнем кластере из девяти сигналов, который наблюдается по- 
сле достижения максимальной нагрузки $F_{\max }$, все сигналы принадлежат магистральному сету. Все кластеры сигналов, за исключением последнего, характеризуются широким диапазоном амплитуд сигналов ЭХЭ, которые могут отличаться в разы (в 6 раз в примере на рис. 2). В то же время в последнем кластере амплитуда сигналов приблизительно одинакова (разброс обычно не превышает $10 \%$ ). Поэтому кластер из десятка почти одинаковых высокоамплитудных сигналов можно рассматривать как наиболее вероятный предвестник потери глобальной устойчивости - образования шейки и разрыва образца.

Можно сказать, что прерывистая кривая растяжения, сигналы дискретной ЭХЭ и составляющие их временной структуры являются одномерными отображениями сложной пространственно-временной структуры макроскопически локализованной пластической деформации на одну степень свободы - временные ряды $\sigma(t)$ и $\Delta E(t)$. В частности, магистральный сет сигналов ЭХЭ является отображением структуры деформационных полос ПЛШ, проходящих или остановившихся в сечении, через которое пройдет магистральная трещина.

Как установлено в предыдущей работе [11], при прерывистой ползучести передний фронт скачка электродного потенциала деформируемого сплава АМг6 сигнала ЭХЭ, соответствует зарождению и расширению деформационной полосы и обусловлен разрушением защитной оксидной пленки $\mathrm{Al}_{2} \mathrm{O}_{3}$ из-за выхода на поверхность сплава дислокаций деформационной полосы, а спад сигнала ЭХЭ - с восстановлением оксида на соответствующем участке поверхности. Дистиллированная вода, как известно, является агрессивной (коррозионной) средой по отношению к неокисленному алюминию [12]: она активно растворяет алюминий в области выхода на поверхность деформационной полосы (анодный процесс). Поэтому после разрыва оксидной пленки вблизи свежеобразованной поверхности формируется двойной электрический слой из гидратированных ионов $\mathrm{Al}^{3+}$ со стороны водного раствора и соответствующего избытка электронов со стороны металла, который приобретает отрицательный заряд, что и вызывает скачок электродного потенциала в отрицательную сторону - сигнал ЭХЭ.

Синхронность процессов формирования деформационной полосы ПЛШ и переднего фронта дискретного сигнала ЭХЭ подтверждается и при растяжении сплава АМг6 в условиях проявления эффекта ПЛШ в специальной серии экспериментов, в которой одновременно с измерением электрохимического отклика проводили высокоскоростную видеосъемку поверхности со скоростью $20000 \mathrm{frames} / \mathrm{s}$. На рис. 3 представлены записи скачка разгрузки $\sigma(t)$, единичного сигнала ЭХЭ $\Delta E(t)$ и временной зависимости площади полосы $A(t)$, т.е. площади, заключенной между границами полосы.

Эволюция полосы ПЛШ, как следует из данных видеосъемки, состоит из двух стадий: стадии очень быстрого прорастания зародыша полосы со скоростью

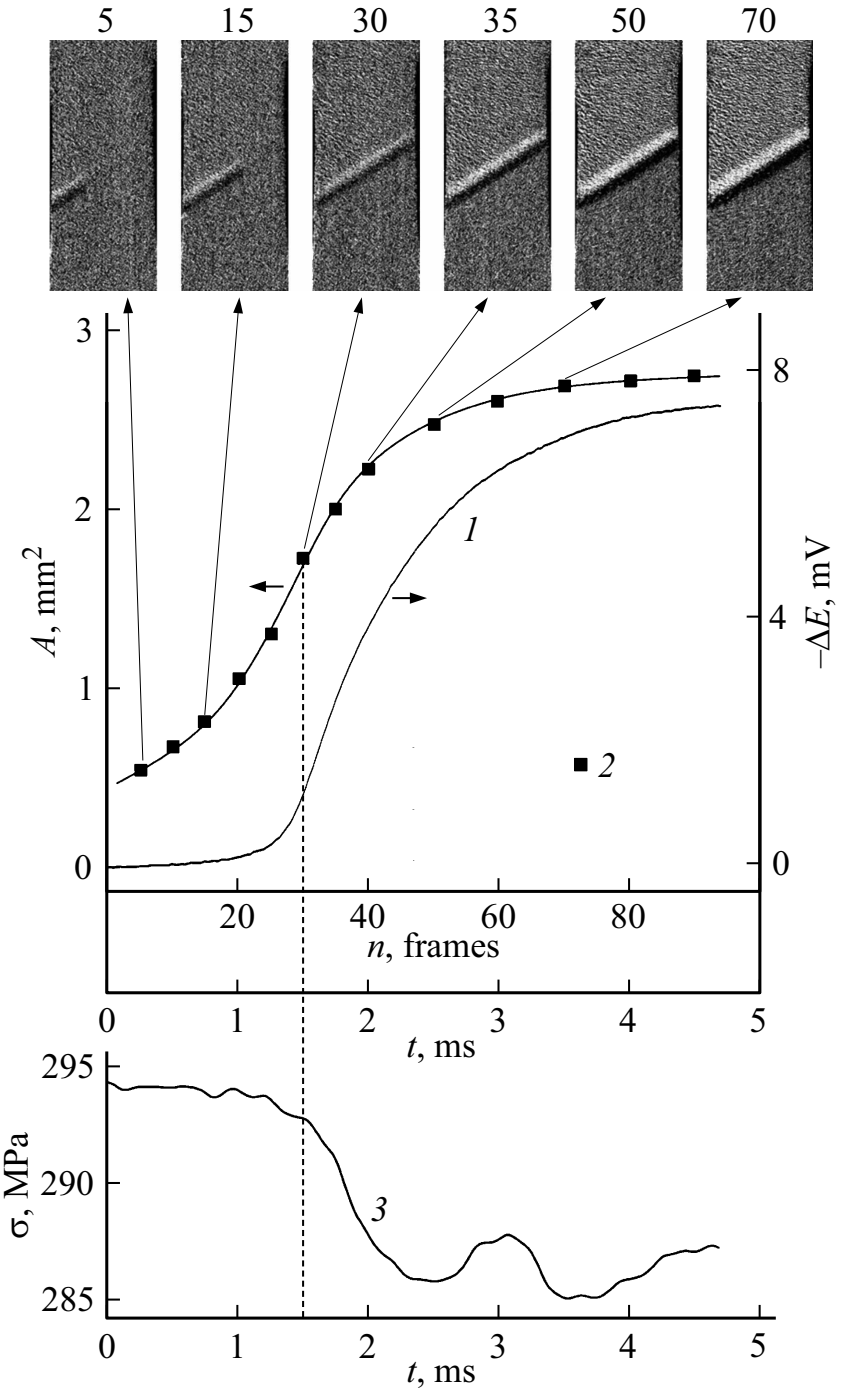

Рис. 3. Корреляция формы фронта сигнала ЭХЭ $\Delta E$ (1), временной зависимости площади полосы $A$ (2) и скачка напряжения $\sigma(3)$. На вставке - результаты компьютерной обработки данных видеосъемки зарождения и расширения полосы ПЛШ. Скорость съемки 20000 frames/s.

его вершины $v_{t} \approx 3-5 \mathrm{~m} / \mathrm{s}$ в направлении, составляющем угол 58-62 ${ }^{\circ}$ к оси растяжения, и последующей стадии расширения полосы со средней скоростью границ полосы в направлении оси растяжения $v_{s}$ около $10 \mathrm{~cm} / \mathrm{s}$ (вставка на рис. 3). Когда ширина полосы достигает величины, сопоставимой с толщиной плоского образца, т. е. около $0.5 \mathrm{~mm}$, скорость расширения полосы $v_{s}$ резко падает и на границе полосы зарождается новая полоса в соответствии с каскадным механизмом размножения полос, описанным в [13]. Анализ форм электрохимического сигнала $\Delta E$ и $A(t)$ показывает весьма высокую корреляцию этих зависимостей с коэффициентом корреляции, равным 0.997 (рассчитанного с помощью программы MathLab) на стадии расширения полосы ПЛШ. Вместе с тем, стадия прорастания зародыша полосы сопровож- 
дается небольшим изменением сигнала, составляющего около $10 \%$ амплитуды сигнала ЭХЭ.

Таким образом, рост абсолютной величины сигнала ЭХЭ обусловлен в основном расширением деформационной полосы, что, как известно, связано с массовым выходом дислокаций на поверхность в ходе развития пластической неустойчивости. После „простреливания“ зародыша полосы через поперечное сечение одна макроскопическая часть образца смещается относительно другой по полосе деформации в плоскости максимальных касательных напряжений. (Для изотропного пластически деформируемого твердого тела эта плоскость составляет угол $55^{\circ} 44^{\prime}$ к оси растяжения [14]). В результате разрыва хрупкой оксидной пленки и образования поверхностных ступенек обнажается ювенильная поверхность алюминиевого сплава. Этот вывод подтверждается данными сканирующей электронной микроскопии участка выхода полосы деформации на поверхность образца (см. рис. 6 в работе [11]). Можно сказать, что амплитуда дискретного сигнала ЭХЭ $\Delta E_{m}$ является мерой локализации пластической деформации алюминиевого сплава, деформируемого в водной среде.

\section{2. Статистика сигналов ЭХЭ и полос деформации плш}

Амплитудное распределение сигналов ЭХЭ исследовалось посредством построения статистической функции распределения $D(s)=N^{-1} d N / d s$ амплитуд дискретных сигналов ЭХЭ $s=\Delta E_{m}$ (рис. 4). В двойных логарифмических координатах эта функция является приблизительно линейной с коэффициентом наклона к $s$-оси, равным -0.78 (вставка на рис. 4 ); здесь $N-$ общее количество сигналов ЭХЭ $d N-$ количество сигналов, амплитуда которых попадает в узкий интервал $s-\delta s / 2$, $s+\delta s / 2$ (см. [15]). Это означает, что амплитудное распределения электрохимических сигналов подчиняется степенному закону $D(s) \sim s^{-\alpha}$ с показателем степени $\alpha=0.78$. Известно, что степенное распределение амплитуд лавин с показателем порядка единицы характерно для землетрясений [16] и является одним из признаков состояния самоорганизующейся критичности (СОК) [17].

Согласно $[17,18]$, для систем с СОК глобальная динамика контролируется дальнодействующими корреляциями между большим числом локальных объектов, в данном случае деформационных полос - источников сигналов ЭХЭ. Пространственная связь, обеспечивающая такую корреляцию, связана с каскадным механизмом размножения деформационных полос, когда каждая полоса, за исключением первичной, зарождается на границе предшествующей полосы [13]. Корреляционным полем являются внутренние напряжения на границе остановившейся полосы и дальнодействующие напряжения изгиба, возникающие вследствие того факта, что отдельные полосы переносят механический заряд источник дальнодействующих напряжений $[13,19]$.

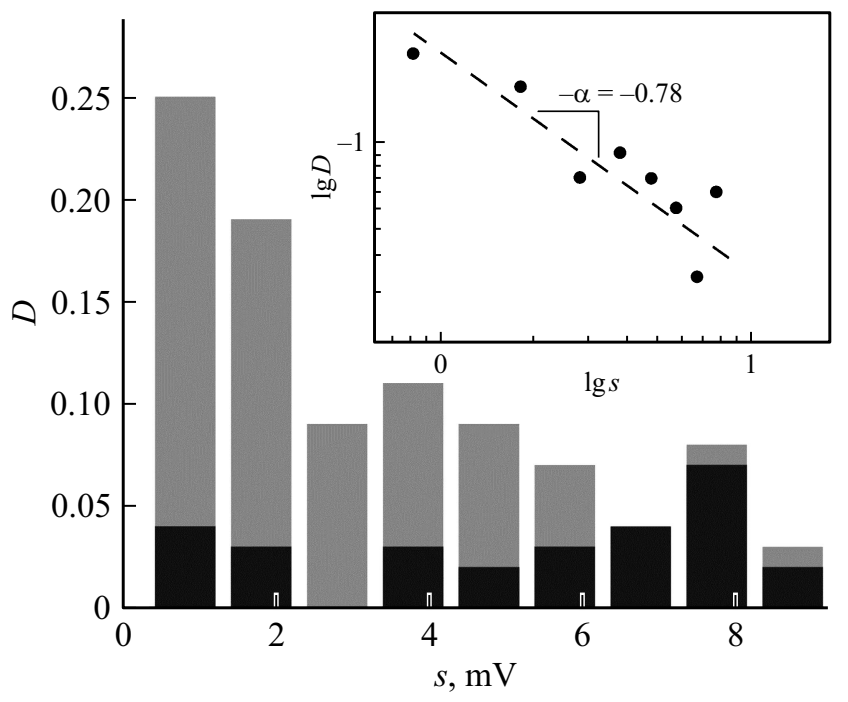

Рис. 4. Статистическая функция распределения $D(s)$ амплитуд $s=\Delta E_{m}$ сигналов ЭХЭ, сопровождающих прерывистую деформацию сплава АМг6 в дистиллированной воде. На вставке - функция $D(s)$ в двойных логарифмических координатах. Прерывистая линия показывает отрицательный наклон линейной аппроксимации с показателем $\alpha=0.78$. Черными столбцами выделено амплитудное распределение магистрального сета сигналов ЭХЭ.

Магистральный сет сигналов ЭХЭ, однако, демонстрирует иное амплитудное распределением, в котором наблюдается рост доли высокоамплитудных сигналов, особенно сигналов, непосредственно предшествующих разрушению образца. (На рис. 4 амплитудное распределение магистрального сета выделено черными столбцами.) Таким образом, в ходе деформирования образца вблизи магистрального сечения происходит автолокализация деформационных полос, генерирующих сигналы ЭХЭ с возрастающей амплитудой.

Рассмотрим теперь особенности пространственного распределения деформационных полос вдоль оси $x$ растяжения образца. Для его построения рабочая часть образца разделялась на эквидистантные нормальные сечения $x=x_{i}$ с шагом $\Delta x=x_{i+1}-x_{i}=0.5 \mathrm{~mm}$, приблизительно равным конечной ширине полосы. Сечение $x_{0}=0$ соответствует „магистральному“ сечению, через которое пройдет магистральная трещина. Далее подсчитывалось количество границ полос, пересекающих каждое сечение, и строилась гистограмма $\Delta N\left(x_{i}\right)$. Типичная гистограмма полос имеет характерный максимум вблизи магистрального сечения (рис. $5, a$ ), т.е. магистральная трещина проходит через сечение, наиболее интенсивно „обработанное“ деформационными полосами, что согласуется с результатами работ $[7,9,20]$.

Синхронные с видеосъемкой поверхности измерения электрохимического и силового откликов показывают, что с ростом деформации растут средняя амплитуда сигналов ЭХЭ и средняя амплитуда скачков напряжения (рис. 1), причем полосы деформации, генерирующие 

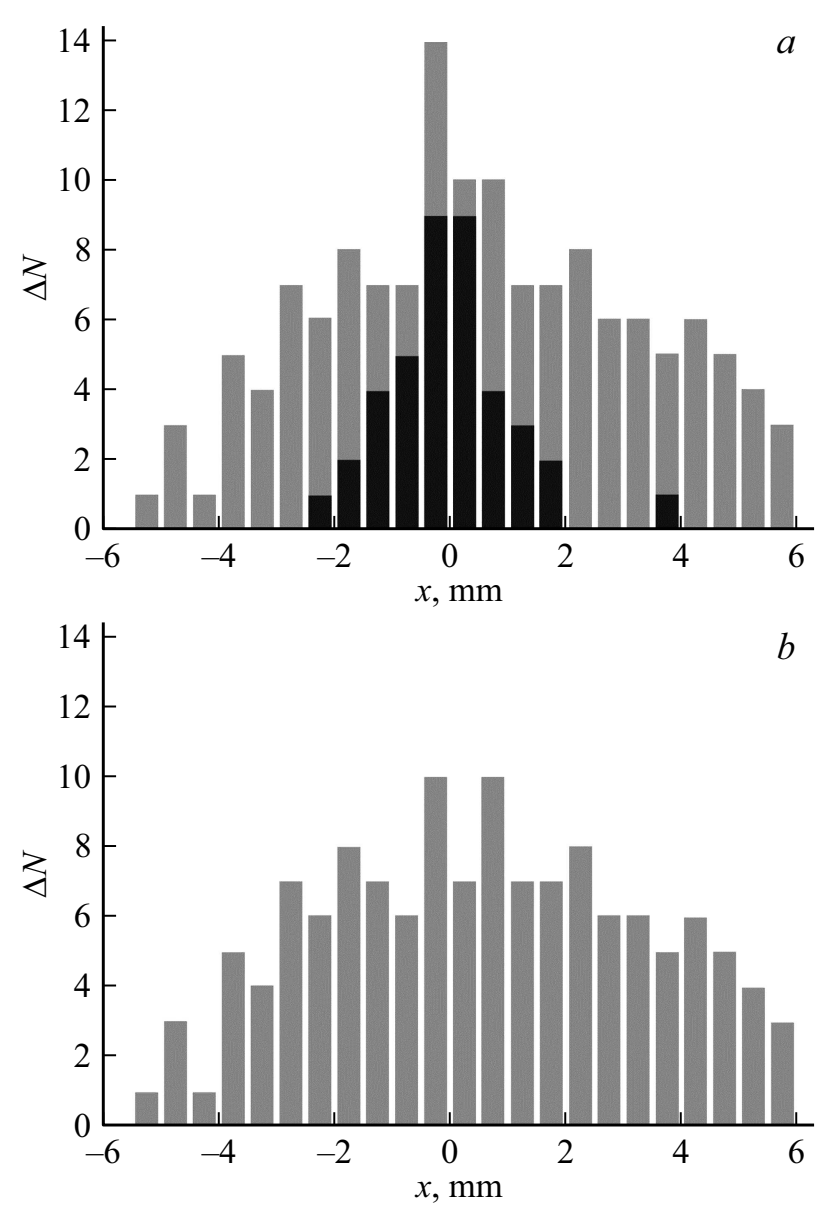

Рис. 5. Гистограмма полос ПЛШ вдоль оси $x$ растяжения образца АМг6: $a$ - от критической деформации $\varepsilon_{c}$ до разрушения (черным цветом выделена гистограмма полос, которые генерируют сигналы ЭХЭ амплитудой больше $\left.0.7 \Delta E_{\max }\right) ; b-$ от $\varepsilon_{c}$ до деформации, соответствующей максимальной приложенной нагрузке $F_{\max }$.

сигналы ЭХЭ с возрастающей амплитудой, постепенно автолокализуются в центральной части образца, в области, прилегающей к магистральному сечению. Для демонстрации этой тенденции на рис. 5, $a$ черным цветом выделена гистограмма полос, которые генерируют сигналы ЭХЭ амплитудой больше $0.7 \Delta E_{\max }$. Огибающая этой гистограммы имеет вид гауссианы с вершиной в магистральном сечении $x_{0}=0$ и полушириной около $2 \mathrm{~mm}$.

Важно подчеркнуть, что после удаления из полной статистической выборки последней серии полос, которые формируются после достижения максимальной нагрузки $F_{\max }$, из гистограммы полос исчезает максимальный выброс вблизи магистрального сечения $x_{0}=0$ (рис. 5, b). Следовательно, эти полосы оказывают наибольшее влияние на разрушение образца. В нашем примере это девять последних полос, возникающих в последние десять секунд до разрушения. Все генерируемые ими сигналы ЭХЭ принадлежат магистральному сету и имеют максимальные амплитуды. Соответству- ющие скачки разгрузки следуют через приблизительно одинаковые временные интервалы и также имеют максимальные амплитуды на прерывистой кривой растяжения.

Отметим, что, согласно общепринятой классификации пластических неустойчивостей ПЛШ [21], при низких скоростях деформации возникают высокоамплитудные скачки разгрузки типа $\mathrm{C}$, которым соответствуют статические полосы деформации; с ростом скорости деформации происходит переход к скачкам нагрузки типа В, которым соответствуют „прыгающие“ полосы деформации, и, наконец, при высоких скоростях деформации наблюдаются низкоамплитудные скачки типа А с выбросом в положительную сторону, эти скачки сопровождаются непрерывным распространением полос ПЛШ типа А.

На большей части прерывистой кривой деформации сплава АМг6 наблюдаются скачки типа В. При больших степенях деформации истинная скорость деформации уменьшается как $\dot{\varepsilon}_{t r}=\dot{\varepsilon}_{0} /(1+\varepsilon)$ и происходит переход от скачков типа В к скачкам типа С. Последние характерны для завершающей стадии деформирования. В частности, последние девять скачков после достижения максимальной нагрузки $F_{\max }$ по внешнем признакам относятся к высокоамплитудным скачкам типа С. Но в отличие от обычных скачков типа $\mathrm{C}$, возникающих вследствие зарождения статических полос в случайных позициях на поверхности образца, девять последних скачков разгрузки возникают в результате формирования статических деформационных полос только в магистральном сечении. Как показано ниже, их динамика и морфология определяют особенности формирования шейки в материале, демонстрирующем прерывистую деформацию ПЛШ.

\section{3. Стадия предразрушения для металла, демонстрирующего эффект ПЛШ}

Известно, что для металлов, демонстрирующих однородное и монотонное пластическое течение (без скачков напряжения и деформационных полос), начало образования шейки соответствует условию Консиде́ре

$$
\frac{d \sigma_{t r}}{d \varepsilon_{t r}}=\sigma_{t r},
$$

где $\sigma_{t r}$ и $\varepsilon_{t r}-$ истинное напряжение и истинная деформация соответственно, которое соблюдается в момент достижения максимальной нагрузки, $F_{\max }$ [22-24]. Справедливо ли это условие начала образования шейки для металлов и сплавов, демонстрирующих эффект Портевена-Ле Шателье, представляет в настоящее время дискуссионный вопрос; альтернативные точки зрения представлены в работах [9 и 25].

Для анализа условия образования шейки в исследуемом сплаве АМг6, демонстрирующем ярко выраженную прерывистую деформацию ПЛШ при комнатной температуре, прерывистую часть кривой растяжения $\sigma_{t r}-\varepsilon_{t r}$ (рис. 6, кривая 1) сглаживали путем 


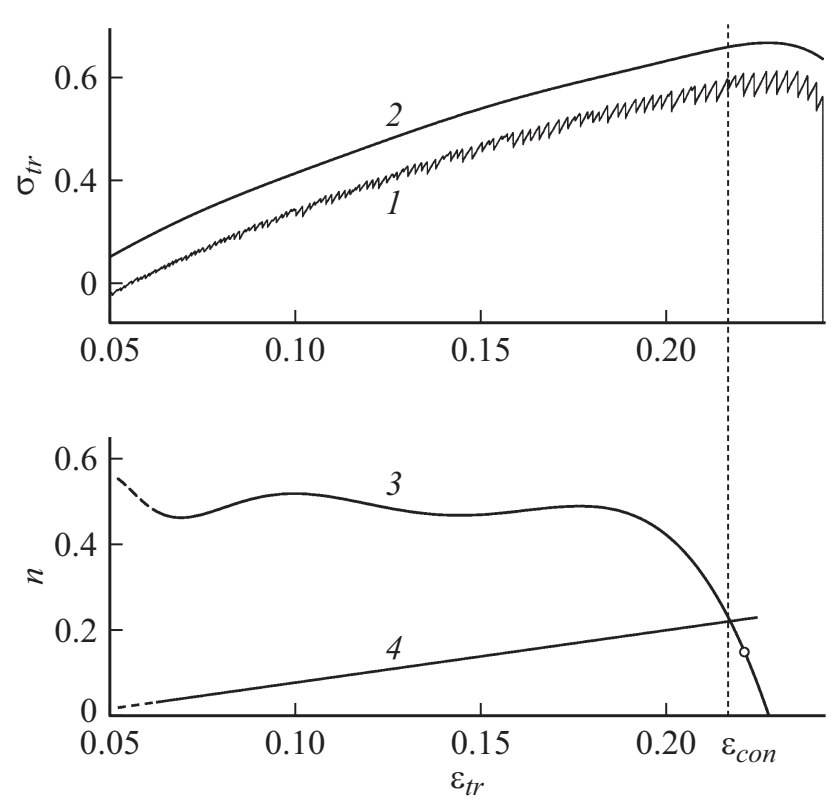

Рис. 6. Результаты обработки деформационной кривой сплава АМг6, представленной на рис. 1: 1 - прерывистая кривая растяжения в координатах $\sigma_{t r}-\varepsilon_{t r}, 2$ - осредненная кривая растяжения, построенная путем аппроксимации кривой 1 полиномом 7-го порядка, 3 - зависимость $n\left(\varepsilon_{t r}\right)$, полученная дифференцированием кривой $2,4-$ линейная зависимость $n=\varepsilon_{t r}$. $\varepsilon_{\text {con }}=0.2170-$ деформация Консидере. Точка обозначает деформацию при максимальной нагрузке $\varepsilon_{t r}\left(F_{\max }\right)=0.2209$. Кривая 2 смещена на $40 \mathrm{MPa} \mathrm{по} \mathrm{оси} \sigma_{t r}$.

ее аппроксимации полиномом седьмого порядка (кривая 2). Полагая, для больших деформаций степенной закон упрочнения $\sigma_{t r}=k \varepsilon_{t r}^{n}$, где $k-$ коэффициент прочности [26], рассчитывали зависимость показателя степени $n=d \ln \sigma_{t r} / d \ln \varepsilon_{t r}$ от истинной деформации $\varepsilon_{t r}$ (кривая 3). Точка пересечения кривой $n\left(\varepsilon_{t r}\right)$ с прямой линией $n=\varepsilon_{t r}$ соответствует деформации Консидере $\varepsilon_{\text {con }}=n=0.2170$. Вместе с тем, истинная деформация, соответствующая максимальной нагрузке, равна $\varepsilon_{t r}\left(F_{\max }\right)=0.2209$ (эта деформация отмечена точкой на рис. 6). Как видно, для осредненной кривой растяжения (кривая 2) условие Консидере достигается несколько раньше достижения максимальной нагрузки на прерывистой кривой растяжения. Разница деформаций $\varepsilon_{c o n}-\varepsilon_{t r}\left(F_{\max }\right)=0.0039$ составляет $1.8 \% \varepsilon_{\text {con }}$ для примера, представленного на рис. 6.

Для материалов, не проявляющих эффект ПЛШ, как известно, деформация Консидере в точности совпадает с деформацией, при которой приложенная нагрузка максимальна [23]. Таким образом, можно считать, что условие Консидере (1) начала образования шейки приблизительно с точностью $\sim 1 \%$ соблюдается и для материалов, демонстрирующих эффект ПЛШ, что согласуется с выводами работы [9], полученными также на примере алюминий-магниевого сплава. Однако процесс формирования шейки в сплаве АМг6 носит дискретный характер, а не непрерывный как для материалов с гладкими деформационными кривыми. На каждом скачке разгрузки, длительностью около 1-3 ms, формируется статическая полоса, проходящая через магистральное сечение. В паузах между скачками продолжительностью $\sim 1 \mathrm{~s}$ материал упрочняется и процесс образования шейки блокируется, а возобновляется только на фронте последующего скачка разгрузки.

Из рис. 6 видно, что после достижения деформации Консидере $\varepsilon_{c o n}$ происходит десять скачков деформации на кривой растяжения, включая последний с разрывом образца, а из рис. 1 следует, что после достижения максимальной нагрузки $F_{\max }$ происходит девять скачков напряжения, т.е. один скачок напряжения оказывается „спорным“, так как он попадает в отмеченный выше интервал деформации $\varepsilon_{c o n}<\varepsilon_{t r}<\varepsilon_{t r}\left(F_{\max }\right)$. Видеосьемка показывает, что этому „спорному“ скачку соответствует полоса деформации, не проходящая через магистральное сечение в отличие от девяти последующих скачков, каждому из которых соответствуют полосы, проходящие через магистральное сечение и участвующие в формировании шейки. Поэтому, как и предполагалось, кластер из девяти последних сигналов ЭХЭ, отвечающих за формирование девяти последних деформационных полос после достижения условия Консидере, является электрохимическим предвестником процесса образования шейки и разрушения образца.

\section{4. Электрохимический предвестник формирования шейки и разрушения образца}

Фрагмент записи электрохимического сигнала $\Delta E(t)$ после деформации Консидере $\varepsilon_{\text {con }}=n$ до разрыва образца вместе с соответствующим фрагментом видеосъемки со скоростью 500 frames/s поверхности представлена на рис. 7. Как видно из рисунка, каждому из девяти сигналов ЭХЭ, образующих последний кластер сигналов предвестник разрушения, - соответствует формирование девяти деформационных полос, проходящих через магистральное сечение. Семь последовательных скачков разгрузки и соответственно дискретных сигналов ЭХЭ возникают в результате формирования трех статичных полос в плоскости максимальных касательных напряжений, составляющих на фронтальной поверхности плоского образца угол около $60^{\circ}$, а на боковой угол 90 четырех сопряженных полос 2-го типа, составляющих на фронтальной поверхности угол около $120^{\circ}$ к оси растяжения (рис. 7, $b$, кадры 4209-7654). Они образуют крестообразную структуру полос (рис. 7,c), аналогичную наблюдаемой на стадии предразрушения в материалах, не демонстрирующих эффект ПЛШ [4-6]. Отметим, что полоса деформации содержит избыток дислокаций одного механического знака $[13,19]$, и рост полосы создает в материале изгибающий момент. Движущей силой формирования сопряженной полосы деформации является релаксация изгибающего момента и связанная 
с ним релаксация суммарного вектора Бюргерса дислокационного ансамбля в деформируемом образце [8].

По мере приближения к моменту разрыва образца углы сопряженных полос приближаются в пределах около $10^{\circ}$ к нормальному сечению и составляют: на 4-м скачке от разрыва $117^{\circ}$, а на 3 -м скачке - $69^{\circ}$ к оси растяжения соответственно. На предпоследнем скачке в области пересечения сопряженных полос образуются сначала полоса локализованного сдвига в плоскости, составляющей угол $90^{\circ}$ к оси растяжения на фронтальной поверхности, и около $60^{\circ}$ на боковой поверхности образца (полоса 1-го типа, кадр 8228), на границе которой зарождается трещина. На последнем скачке (кадр 8703) она перерастает в магистральную трещину, которая распространяется по границе полосы 1-го типа и представляет трещину поперечного сдвига [23].
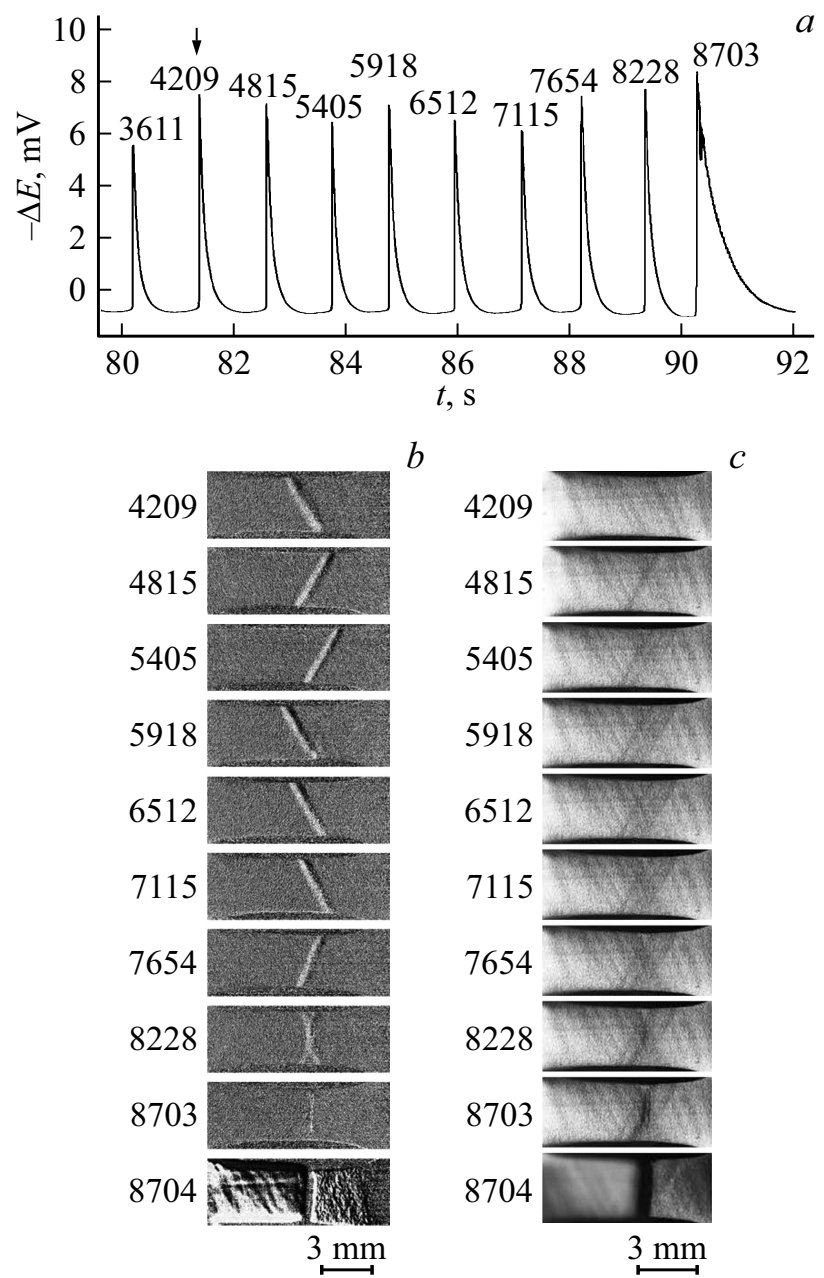

Рис. 7. Временные и видеоряды на стадии предразрушения: $a-$ фрагмент сигнала ЭХЭ на стадии образования шейки; $b$ и $c$ - соответствующие этому фрагменту данные видеосъемки локализации деформационных полос вблизи магистрального сечения; $b-$ обработанные компьютерной программой вычитания последовательных изображений; $c$ - необработанные кадры видеосъемки. Числа - номера кадров видеосъемки со скоростью $500 \mathrm{frames} / \mathrm{s}$. Стрелкой отмечен момент достижения максимальной нагрузки $F_{\max }$.

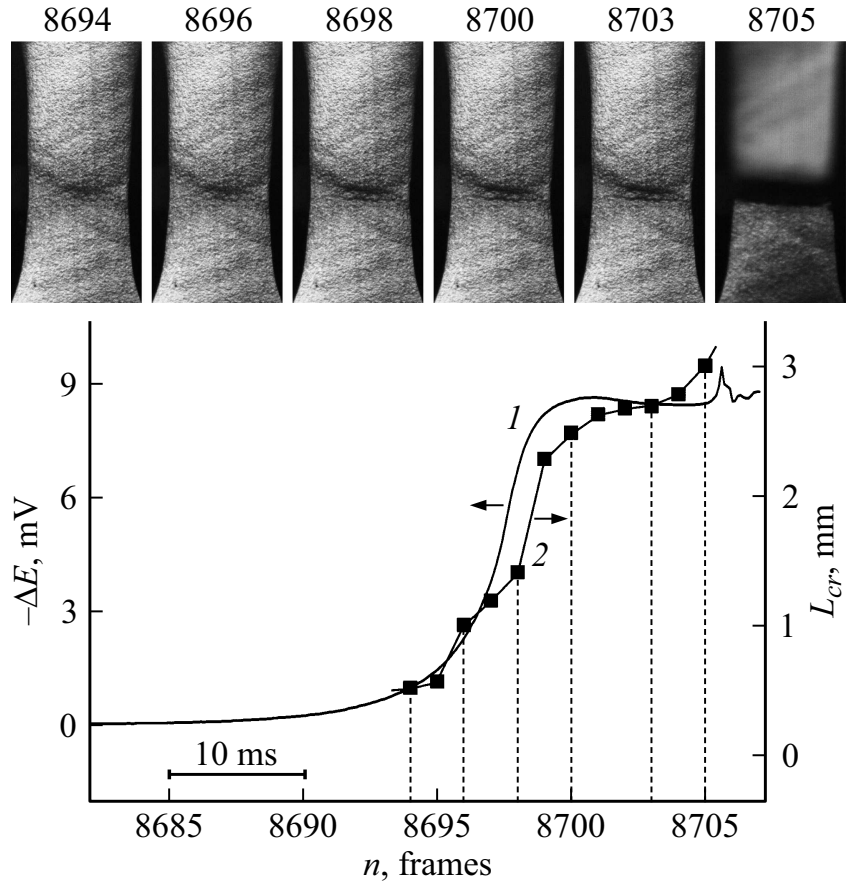

Рис. 8. Корреляция формы фронта сигнала ЭХЭ $\Delta E$ (1) и длины трещины $L_{c r}(2)$. На вставке - данные видеосъемки поверхности за $20 \mathrm{~ms}$ до разрушения образца. Числа $n-$ номера кадров видеосъемки. Скорость съемки 500 frames/s. Вертикальными линиями отмечены точки графика $L_{c r}(2)$, соответствующие номерам кадров видеосъемки.

Форма сигнала ЭХЭ в последние $20 \mathrm{~ms}$ до разрыва образца (рис. 8) отражает основные особенности завершающей стадии формирования шейки: рост абсолютной величины сигнала, отвечающий прорастанию трещины по границе полосы 1-го типа в структуре шейки (рис. 8, кадры 8694-8700), последующее плато длительностью $\sim 10 \mathrm{~ms}$, соответствующее остановке трещины, и особенность продолжительностью менее $\sim 1 \mathrm{~ms}$ в момент разрыва образца (кадр 8705).

Таким образом, измерение электрохимической эмиссии позволяет по структуре дискретных сигналов ЭХЭ выявлять стадию предразрушения алюминиевого сплава, демонстрирующего прерывистую деформацию ПЛШ, что может представлять ценность для мониторинга поверхности деформируемого в водной среде сплава в ситуациях, когда использование традиционных методов (оптических, акустических или тензометрических) затруднено или невозможно.

\section{Заключение}

Динамика деформационных полос Портевена-Ле Шателье и разрушение алюминий-магниевого сплава АМг6, деформируемого в дистиллированной воде, исследовалось высокоскоростными методами, включая метод электрохимической эмиссии. Показано, что дискретные сиг- 
налы электрохимической эмиссии - скачки электродного потенциала деформируемого сплава, - являются отображением сложной пространственно-временной структуры деформационных полос на одну степень свободы. Выявлен „магистральный“ сет сигналов совокупность сигналов ЭХЭ, отображающая структуру деформационных полос ПЛШ, остановившихся или проходящих через сечение образца, через которое пройдет магистральная трещина. Такая структура полос: a) спонтанно формируется в течение всей стадии прерывистой деформации; б) генерирует дискретные сигналы ЭХЭ с возрастающей амплитудой; в) включает в себя геометрически сопряженные полосы деформации, которые образуются для компенсации изгибающего момента, вызванного эволюцией отдельной полосы с избытком дислокаций одного механического знака. В ходе прерывистого течения после достижения деформации Консидере сопряженные полосы ПЛШ постепенно с каждым последующим скачком напряжения формируют крестообразную структуру локализованной деформации шейки, в центре которой зарождается магистральная трещина. Выявлен „электрохимический предвестник“ разрушения сплава АМг6 в водной среде - серия сигналов ЭХЭ, отображающая дискретный характер формирования шейки. Форма фронта последнего сигнала ЭХЭ отражает кинетику подрастания трещины до момента разрыва образца.

Сигнал ЭХЭ, таким образом, является полезным физическим инструментом непрерывного мониторинга деформационных полос и трещин в алюминиевом сплаве, деформируемом в водной среде, который позволяет считывать количество образующихся полос, фиксировать моменты зарождения полос, проводить их статистический и корреляционный анализ, регистрировать формирование последнего кластера дискретных сигналов ЭХЭ - предвестника „катастрофы“ - образования шейки и разрыва образца. Полученные результаты могут быть использованы для разработки технологии мониторинга зон локализации деформации и трещин в конструкциях или изделиях из алюминиевых сплавов, которые эксплуатируются в водных средах.

\section{Финансирование работы}

Высокоскоростные in situ исследования динамики деформационных полос и трещин выполнены при частичной поддержке РНФ (проект № 18-19-00304), электрохимические измерения в агрессивной среде выполнены в рамках госзадания Минобрнауки РФ № 3.8515.2017/8.9 с использованием оборудования ЦКП ТГУ им. Г.Р. Державина, а статистический анализ деформационных полос и сигналов электрохимической эмиссии выполнен при поддержке РФФИ (проект № 19-08-00395).

\section{Конфликт интересов}

Авторы заявляют, что у них нет конфликта интересов.

\section{Список литературы}

[1] Степанов В.А. Основы пластической прочности кристаллов. М.: Наука, 1974. 235 с.

[2] Halim H., Wilkinson D.S., Niewczas M. // Acta Mater. 2007. Vol. 55. P. 4151-4160. DOI: 10.1016/j.actamat.2007.03.007

[3] Spencer K., Corbin S.F., Lloyd D.J. // Mater. Sci. Eng. 2002. Vol. A 325. N 1-2. P. 394-404. DOI: $10.1016 / \mathrm{S} 0921-5093(01) 01481-2$

[4] Панин В.Е., Деревягина Л.С., Дерюгин Е.Е. идр. // Физ. мезомех. 2003. Т. 6. Вып. 6. С. 97-106.

[5] Панин В.Е., Гриняев Ю.В. // Физ. мезомех. 2003. Т. 6. Вып. 4. С. 9-36.

[6] Деревягина Л.С., Панин В.Е., Гордиенко А.И. // Физ. мезомех. 2007. Т. 10. Вып. 4. С. 59-72.

[7] Шибков А.А., Золотов А.Е., Желтов М.А., Шуклинов А.В., Денисов А.А. // ФТТ. 2011. Т. 53. Вып. 10. С. 1873-1878. [Shibkov A.A., Zolotov A.E., Zheltov M.A., Shuklinov A.V., Denisov A.A. // Phys. Solid State. 2011. Vol. 53. N 10. P. 1975-1980.]

[8] Шибков А.А., Желтов М.А., Золотов А.Е., Денисов А.А. // ФТТ. 2011. Т. 53. Вып. 10. С. 1879-1884. [Shibkov A.A., Zheltov M.A., Zolotov A.E., Denisov A.A. // Phys. Solid State. 2011. Vol. 53 N 10. P. 1981-1986.]

[9] Yuzbekova D., Mogucheva A., Zhemchuzhnikova D., Lebedkina T., Lebyodkin M., Kaibyshev R. // Intern. J. Plasticity. 2017. Vol. 96. P. 210-226. DOI: $10.1016 /$ j.ijplas.2017.05.004

[10] Шибков А.А., Денисов А.А., Гасанов М.Ф., Золотов А.Е., Желтов М.А. // ФТТ. 2019. Т. 61. Вып. 2. С. 296-301. DOI: $10.21883 /$ FTT.2019.02.47129.224

[11] Шибков А.А., Денисов А.А., Гасанов М.Ф., Золотов А.Е., Желтов М.A. // Кристаллография. 2019. Т. 64. Вып. 3 (в печати).

[12] Vargel Ch. Corrosion of aluminum. Elsevier Ltd., 2004. 658 p.

[13] Шибков А.А., Золотов А.Е., Михлик Д.В., Желтов М.А., Шуклинов А.В., Аверков В.А., Денисов А.А. // Деформация и разрушение материалов. 2009. Вып. 8. С. 23-30.

[14] Хилл Р. Математическая теория пластичности. М.: Гостехиздат, 1956. $407 \mathrm{c}$.

[15] Lebedkina T.A., Lebyodkin M.A. // Acta Mater. 2008. Vol. 56. P. 5567-5574. DOI: 10.1016/j.actamat.2008.07.025

[16] Gutenberg B., Richter C.F. // Ann. di Geophisica. 1956. Vol. 9. P. 1-15.

[17] Jensen H.J. Self-organized criticality/Cambridge University Press, Cambridge. 1998.

[18] Bak P., Tang C., Wiessenfeld K. // Phys. Rev. A. 1988. Vol. 38. P. 364-374. DOI: 10.1103/PhysRevA.38.364

[19] Шибков А.А., Золотов А.Е., Михлик Д.В., Желтов М.А., Шуклинов А.В.// Деформация и разрушение материалов. 2009. Вып. 9. С. 22-29.

[20] Шибков А.А., Желтов М.А., Гасанов М.Ф., Золотов A.E. // ФТТ. 2018. Т. 60. Вып. 2. С. 315-322. DOI: $10.21883 /$ FTT.2018.02.45386.158

[21] Chihab K., Estrin Y., Kubin L.P., Vergnol J. // Scripta Metall. 1987. Vol. 21. P. 203-208. DOI: $10.1016 / 0036-9748(87) 90435-2$

[22] Хоникомб Р. Пластическая деформация металлов. М.: Мир, 1972. $191 \mathrm{c.}$

[23] Владимиров В.И. Физическая природа разрушения металлов. М.: Металлургия, 1984. 280 с. 
[24] Малыгин Г.А. // ФТТ. 2005. Т. 47. Вып. 2. С. 236-241. [Malygin G.A. // Phys. Solid State. 2005. Vol. 47. N 2. P. 246-251.]

[25] Kang J., Wilkinson D.S., Jain M., Embury J.D., Beaudoin A.J., Kim S., Mishira R., Sachdev A.K. // Acta Material. 2006. Vol. 54. P. 209-218. DOI: $10.1016 /$ j.actamat.2005.08.045

[26] Marciniak Z., Duncan J.L., Hu S.J. Mechanics of sheet metal forming (2nd ed.), Oxford: Butterworth-Heinemann, 2002. $211 \mathrm{p}$.

[27] Шибков А.А., Золотов А.Е., Желтов М.А., Денисов А.А., Гасанов М.Ф., Кочегаров С.С. // ЖТФ. 2016. Т. 86. Вып. 5. C. 68-76. [Shibkov A.A., Zolotov A.E., Zheltov M.A., Denisov A.A., Gasanov M.F., Kochegarov S.S. // Tech. Phys. 2016. Vol. 61. N 5. P. 707-714.] 